\title{
Eficiência produtiva e reprodutiva de vacas de corte Purunã de diferentes categorias
}

\section{Productive and reproductive efficiency of Purunã beef cows of different categories}

\author{
Ivan Cesar Furmann Moura ${ }^{1 *}$; Fernando Kuss²; José Luis Moletta ${ }^{3}$; \\ Luis Fernando Glazenapp de Menezes²; Douglas Sampaio Henrique²; \\ Antônio Altino Cherubin ${ }^{4}$; Michele de Paris ${ }^{5}$
}

\section{Resumo}

O presente trabalho foi desenvolvido na Estação Experimental Fazenda Modelo do Instituto Agronômico do Paraná (IAPAR), município de Ponta Grossa - PR, no período de junho de 2011 a março de 2012. Fêmeas bovinas da raça Purunã $(n=159)$ foram divididas em três categorias conforme segue: vacas jovens (33-36 meses de idade), vacas adultas (43-90 meses de idade) e vacas velhas ( $>90$ meses de idade). $\mathrm{O}$ balanço energético negativo foi evidente em todas as categorias de vacas, com perda de peso acima de $13 \mathrm{~kg}$ até os 60 dias pós-parto. A taxa de prenhez não diferiu significativamente entre as três categorias, tendo sido $93 \%$ para vacas jovens, $94 \%$ para vacas velhas e $96 \%$ para vacas adultas.Vacas adultas desmamaram bezerros com maior peso ao desmame $(221,20 \mathrm{~kg})$ em relação às vacas jovens $(190,00 \mathrm{~kg})$ e velhas $(205,19 \mathrm{~kg})$. As taxas de prenhez do rebanho de cria não sofreram influência da categoria das vacas de corte quando mantidas em boas condições corporais e nutricionais ao parto e durante a estação de monta. Nessas circunstâncias, vacas jovens acasaladas aos dois anos de idade apresentam eficiência produtiva e reprodutiva semelhante a de vacas adultas, enquanto a categoria de vacas velhas apresenta a menor eficiência reprodutiva do plantel de cria.

Palavras-chave: Bezerro, desempenho, desmame, escore corporal, gestação

\begin{abstract}
This study was conducted at Fazenda Modelo an Experimental Estation belonged to the Instituto Agronômico do Paraná. (IAPAR), in the city of Ponta Grossa - PR, in the period from June2011 to March 2012.Purunã bovine females $(n=159)$ class were divided into three categories as follows: young cows (33-36 months old) adult cows (43-90 months old) and older cows (> 90 months). The negative energy balance was evident in all categories of cows with weight loss above $13 \mathrm{~kg}$ to 60 days postpartum. The pregnancy rate did not differ significantly among the three categories were $93 \%$ for young cows, old cows for $94 \%$ and $96 \%$ for adult cows. Adult cows weaned calves with higher weaning weight $(221.20$ $\mathrm{kg}$ ) as compared to young cows (190.00 kilograms) and old (205.19 kg). Rates of the pregnant cow
\end{abstract}

\footnotetext{
${ }^{1}$ Zootecnista, M.e em Zootecnia, Técnico de Pesquisa e Extensão, Laboratório de Mecanização Agrícola, Universidade Estadual de Ponta Grossa, UEPG, Ponta Grossa, PR. E-mail: ivancesar.moura@bol.com.br

${ }^{2}$ Profs. do Programa de Pós Graduação em Zootecnia, Universidade Tecnológica Federal do Paraná, UTFPR, Dois Vizinhos, PR. E-mail: fernandokuss@utfpr.edu.br; luismenezes@utfpr.edu.br; douglas@utfpr.edu.br

${ }^{3}$ Pesquisador, Instituto Agronômico do Paraná, IAPAR, Fazenda Modelo, Ponta Grossa, PR. E-mail: moletta@iapar.br

${ }^{4}$ Médico Veterinário, Monitor de Processo da Unidade Industrial de Carnes Castrolanda, Castro, PR. E-mail: ticopensador@gmail.com

${ }_{5}^{5}$ Discente do Curso de Mestrado do Programa de Pós Graduação em Zootecnia, UTFPR, Dois Vizinhos, PR. E-mail: micheli. deparis@gmail.com

* Autor para correspondência
} 
herd were not influenced by the category of beef cows when maintained in good body condition and nutritional at calving and during the breeding season. In these circumstances, young cows bred at two years of age are similar to productive and reproductive performance of mature cows, while the category of old cows has the lowest reproductive efficiency of breeding squad.

Key words: Calf, performance, weaning, body condition, pregnancy

\section{Introdução}

O aumento das taxas de natalidade e de desmame, diminuição da idade ao primeiro parto e ao abate são premissas básicas para se aumentara eficiência produtiva em rebanhos de corte (OLIVEIRA; BONATO; SANTOS, 2011). Indicadores que fundamentam a eficiência do processo produtivo na fase de cria, como as taxas de prenhez, de natalidade e de desmama, o peso à desmama, a produtividade em quilos de bezerros desmamados/vaca exposta à reprodução e a eficiência por área destinada à cria apresentam baixos índices e continuam pouco estudados nos sistemas de produção (VIEIRA et al., 2005).

Avaliando o desempenho reprodutivo de vacas nelore, Oliveira, Bonato e Santos (2011) concluíram que a taxa de gestação no final da estação de monta foi afetada pela categoria animal, indicando que as primíparas precisam de maior atenção em relação as suas exigências nutricionais, para que sua fertilidade não seja comprometida. Associação negativa entre a idade da vaca e o intervalo entre partos é atribuída à maior perda de peso corporal e escore de condição corporal no pós-parto de vacas primíparas (MORRIS; MOREL; KENYON, 2006; RENQUIST et al., 2006).

A eficiência produtiva em bovinos de corte na fase de cria está diretamente relacionada com o desempenho reprodutivo das fêmeas, com habilidade materna e com o potencial de ganho de peso dos bezerros. Os fatores que afetam o desempenho do rebanho de cria são reprodução (taxa de desmame) e o peso médio dos bezerros ao desmame, determinado pelo peso ao nascer mais o ganho de peso durante o período de aleitamento (CERDÓTES et al., 2004).
Neste sentido, o presente trabalho teve por objetivo estudar o desempenho produtivo e reprodutivo entre as diferentes categorias de vacas de corte, assim como o desempenho das suas progênies durante o período de amamentação.

\section{Material e Métodos}

O experimento foi realizado na Estação Experimental Fazenda Modelo do Instituto Agronômico do Paraná (IAPAR), localizado no município de Ponta Grossa - PR. Conforme a classificação de Köeppen, o clima da região se enquadra na variedade $\mathrm{Cfb}$, temperado sem estação seca, com temperatura média anual de $17,6^{\circ} \mathrm{C}$, média das máximas de $24,3^{\circ} \mathrm{C}$ e média das mínimas de $8,5^{\circ} \mathrm{C}$. A precipitação média anual é de 1.400 $\mathrm{mm}$, sendo as chuvas mais frequentes no período de primavera - verão e mais escassas no outono. $\mathrm{O}$ período experimental foi de junho de 2011 a março de 2012, o qual compreende as estações de nascimento (junho, julho e agosto), monta (setembro, outubro e novembro) e desmame (fevereiro e março).

Os animais utilizados neste trabalho (159 vacas e seus respectivos bezerros) são oriundos de um trabalho realizado para avaliar diferentes sistemas de desmame (desmame precoce, amamentação controlada e desmame convencional).Como não houve interação entre categorias e sistema de desmame, os dados avaliados para a categoria das vacas foram discutidos separadamente no presente trabalho. Os animais utilizados eram da raça Purunã (composto por $40 \%$ de Charolês; $25 \%$ de Aberdeen Angus; $25 \%$ Caracu e 10\% Nelore).

As vacas foram distribuídas em três classificações de categoria conforme a idade ao 
parto: vacas jovens ( $\mathrm{n}=46$, primíparas, com idade até 43 meses); vacas adultas $(\mathrm{n}=54,2$ a 5 partos, com idade entre 44 e 90 meses); e o grupo composto por vacas velhas $(n=59,>6$ partos, vacas com idade superior a 90 meses). Todas as categorias foram mantidas em pastagem de aveia (Avena sativa L.) e triticale (X Triticosecale wittmack) durante o período de estação de nascimento (junho, julho e agosto) até o segundo mês da estação de monta (outubro). Em seguida, vacas e bezerros foram encaminhados para áreas de pastagem predominantes de Hemárthria altíssima e campo nativo, onde permaneceram até o desmame, o qual foi conduzido em duas etapas (fevereiro e março), com o intuito de obter idade média de sete meses ao desmame. Todas as áreas pastoris continham água de boa qualidade e sal mineralizado à vontade.

A distribuição das categorias seguiu o mesmo modelo utilizado para a avaliação do sistema de desmame, sendo divididas em duas etapas, vacas paridas no primeiro mês de estação de monta (junho) e vacas paridas no segundo mês (julho). A terceira etapa com os bezerros nascidos em agosto não ocorreu devido ao baixo número de partos e o desproporcional número de vacas entre as categorias. Mesmo os bezerros sendo submetidos a diferentes sistemas de manejo, o peso ao desmame foi considerado aos sete meses, ocorrendo também em duas etapas, conforme a ordem de parto, sendo pesados no início de fevereiro de 2012 os bezerros nascidos em junho de 2011 e, em março de 2012 os bezerros nascidos em julho de 2011.

As vacas e bezerros foram submetidos a avaliações periódicas aproximadamente a cada 30 dias para o controle do peso e avaliação do escore de condição corporal (ECC) segundo a metodologia de Lowman (1973); sendo 1 = muito magro, 2 = magro, 3 = médio, $4=$ gordo e $5=$ muito gordo. A pontuação de ECC foi dada sempre pelo mesmo avaliador, pelo fato de se ter a mesma calibração visual entre um período de avaliação e outro.

O diagnóstico de gestação das vacas foi realizado
60 dias após o encerramento da estação de monta (final do mês de janeiro), por meio de ultrassonografia transretal, utilizando aparelho da marca Aloka SSD500 com transdutor linear de $5 \mathrm{MHz}$. Durante o diagnóstico de gestação, foi estimada a idade do feto com intervalo aproximado de 23 dias, sendo que no dia da realização do diagnóstico haviam fetos com idade aproximada de 60 a 83 dias; 83 a 106; 106 a 129 e de 129 a 152 dias. Com isso, foi possível estimar os dias para concepção a partir do início da estação de monta, assim como, o intervalo do parto à concepção, pois a cada intervalo de idade do feto (dias) seria o período de concepção da vaca, ou seja: de 0 a 23 dias; 23 a 46; 46 a 69 e 69 a 92 dias a partir do início da estação de monta.

Para a determinação das características de índice de produção de bezerro e eficiência produtiva das vacas seguiu-se o modelo utilizado por Vaz, Lobato e Restle (2010), onde: Índice de produção de bezerros $(\mathrm{kg}$ de bezerro/vaca $)=\left(\mathrm{PBD}^{*} \mathrm{TP}\right) / 100$; Eficiência produtiva ao desmame $=(\mathrm{PBD} / \mathrm{PVD}) * 100 \mathrm{e}$; Eficiência reprodutiva $(\mathrm{kg}$ de bezerro/100kg de vaca $)=(((\mathrm{PBD} / 100) * \mathrm{TP}) * 100) / \mathrm{PVD}$.As siglas PBD, TP, PVD e PVP significam, respectivamente, peso do bezerro ao desmame $(\mathrm{kg})$, taxa de prenhez $(\%)$, peso da vaca ao desmame $(\mathrm{kg})$ e peso da vaca ao parto $(\mathrm{kg})$.

O delineamento experimental utilizado foi o inteiramente casualizado. A análise estatística dos dados foi realizada utilizando o programa Statistical Analysis System (SAS, 2001). As médias de desempenho das vacas e dos bezerros foram comparadas pelo teste de Tukey $(\mathrm{p}=0,05)$. A taxa de prenhez foi analisada pelo teste do qui-quadrado, utilizando o procedimento General Linear Models (GLM), que considera o desbalanceamento do número de observações. Nas análises foi incluído o efeito do sexo dos bezerros como co-variável e interação entre o sexo do bezerro e a categoria da vaca, segundo o modelo:

$$
\mathrm{Y}_{\mathrm{ijk}}=\mu+\mathrm{C}_{\mathrm{i}}+\mathrm{P}_{\mathrm{j}}+\mathrm{S}_{\mathrm{k}}+\left(\mathrm{S}^{*} \mathrm{C}\right)_{\mathrm{ik}}+\mathrm{E}_{\mathrm{ijk}}
$$

Em que: 
$\mathrm{Y}_{\mathrm{ijk}}=$ Variáveis independentes;

$\mu=$ média geral das observações;

$\mathrm{C}_{\mathrm{i}=}$ Efeito da categoria da vaca de ordem "i" sendo 1 = Vacas primíparas; 2 =Vacas adultas e 3 = Vacas velhas;

$\mathrm{P}_{\mathrm{j}}=$ Efeito da etapa dos tratamentos de ordem “j” sendo 1 = bezerros nascidos em Junho e $2=$ bezerros nascidos em julho;

$\mathrm{S}_{\mathrm{k}}=$ efeito do sexo do bezerro de ordem " $\mathrm{k}$ " sendo $1=$ macho e 2 = fêmea;

$\left(C^{*} \mathrm{~S}\right)_{\mathrm{ik}=}$ efeito da interação entre a i-ésima categoria da vacas e o k-ésimo sexo do bezerro;

$\mathrm{E}_{\mathrm{ijk}}=$ erro aleatório associado à cada observação, com distribuição supostamente normal e independente NID $\left(0, \sigma^{2}\right)$.

\section{Resultados e Discussão}

O menor peso observado para as vacas jovens, paridas entre 33 e 36 meses de idade em relação às vacas adultas e velhas, está de acordo com o relatado por Rovira (1996), de que o peso das vacas jovens $(383,89 \mathrm{~kg})$ ao parto deve ser superior a $80 \%$ do peso adulto $(452,35 \mathrm{~kg})$. Com isso é evitado a severa mobilização de reservas corporais para crescimento das vacas jovens, amenizando o balanço energético negativo. Porém, vacas velhas apresentaram maior $(\mathrm{P}<0,05)$ ECC ao parto $(3,56$ pontos $)$ que as vacas adultas (3,35 pontos) e jovens (3,26 pontos, Tabela 1). Este fato deve-se à maturidade das vacas velhas, que proporciona um maior aproveitamento de nutrientes para a deposição de gordura, o que não acontece com animais mais jovens, que ainda se encontram em estágio de crescimento.

Tabela 1. Médias de desempenho, erro padrão, coeficiente de variação $(\mathrm{CV})$ e probabilidade ( $>\mathrm{F})$ para vacas de cria de diferentes categorias.

\begin{tabular}{|c|c|c|c|c|c|}
\hline \multirow{2}{*}{ Características } & \multicolumn{3}{|c|}{ Categoria da vaca } & \multirow{2}{*}{$\mathrm{CV}$} & \multirow{2}{*}{$\operatorname{Pr}>\mathrm{F}$} \\
\hline & Jovens & Adultas & Velhas & & \\
\hline & \multicolumn{3}{|c|}{ Peso $(\mathrm{kg})$} & & \\
\hline Ao parto & $383,89 \pm 6,95^{\mathrm{b}}$ & $444,07 \pm 6,33^{\mathrm{a}}$ & $452,35 \pm 6,10^{\mathrm{a}}$ & 10,50 & $<.0001$ \\
\hline Ao início da estação de monta & $368,21 \pm 7,35^{\mathrm{b}}$ & $431,88 \pm 6,60^{\mathrm{a}}$ & $434,78 \pm 6,35^{\mathrm{a}}$ & 11,53 & $<.0001$ \\
\hline Ao final da estação de monta & $414,43 \pm 7,31^{\mathrm{b}}$ & $471,99 \pm 6,58^{\mathrm{a}}$ & $484,14 \pm 6,41^{\mathrm{a}}$ & 10,29 & $<.0001$ \\
\hline \multirow[t]{2}{*}{ Ao desmame } & $399,40 \pm 7,65^{\mathrm{b}}$ & $451,92 \pm 6,89^{\mathrm{a}}$ & $470,59 \pm 6,71^{\mathrm{a}}$ & 10,87 & $<.0001$ \\
\hline & \multicolumn{3}{|c|}{ Escore de condição corporal (pontos) $)^{1}$} & & \\
\hline Ao parto & $3,26 \pm 0,05^{\mathrm{b}}$ & $3,35 \pm 0,04^{\mathrm{b}}$ & $3,56 \pm 0,04^{\mathrm{a}}$ & 9,28 & 0,002 \\
\hline Ao início da estação de monta & $3,05 \pm 0,05^{\mathrm{b}}$ & $3,17 \pm 0,05^{\mathrm{ab}}$ & $3,24 \pm 0,05^{\mathrm{a}}$ & 10,90 & 0,0476 \\
\hline Ao final da estação de monta & $3,54 \pm 0,05^{\mathrm{b}}$ & $3,64 \pm 0,04^{b}$ & $3,84 \pm 0,04^{\mathrm{a}}$ & 8,35 & 0,0002 \\
\hline \multirow[t]{2}{*}{ Ao desmame } & $3,32 \pm 0,06^{\mathrm{b}}$ & $3,51 \pm 0,06^{\mathrm{b}}$ & $3,83 \pm 0,06^{\mathrm{a}}$ & 11,41 & 0,0001 \\
\hline & \multicolumn{3}{|c|}{ Ganho de peso $(\mathrm{kg})$} & & \\
\hline Do parto ao início da estação de monta & $-15,89$ & $-13,43$ & $-17,57$ & $-206,17$ & 0,7899 \\
\hline Durante a estação de monta & $46,22 \pm 5,43$ & $40,11 \pm 4,89$ & $49,36 \pm 4,76$ & 76,33 & 0,3149 \\
\hline \multirow[t]{2}{*}{ Do final da estação de monta ao desmame } & $-15,03 \pm 3,65$ & $-17,96 \pm 3,34$ & $-13,55 \pm 3,25$ & $-84,98$ & 0,2749 \\
\hline & \multicolumn{3}{|c|}{ Desempenho reprodutivo } & & \\
\hline Dias para concepção na estação de monta & $46,20 \pm 2,80$ & $44,58 \pm 2,49$ & $41,98 \pm 2,47$ & 40,01 & 0,3280 \\
\hline Período parto concepção, dias & $111,37 \pm 3,03$ & $109,86 \pm 2,72$ & $108,25 \pm 2,70$ & 17,29 & 0,7466 \\
\hline Taxa de prenhez, $\%$ & $93 \pm 0,03$ & $96 \pm 0,03$ & $94 \pm 0,03$ & 24,14 & 0,7018 \\
\hline
\end{tabular}

$1=1$ ponto para muito magra e 5 pontos para vaca muito gorda. Médias seguidas por letras diferentes na mesma linha diferem estatisticamente entre si pelo teste de Tukey $(\mathrm{P}<0,05)$.

Fonte: Elaboração dos autores. 
Independente da idade ao parto, todas as categorias apresentaram redução $(\mathrm{P}<0,05)$ de peso e ECC no período entre o parto e o início da estação de monta, evidenciando o fenômeno do balanço energético negativo pós-parto. Durante a estação de monta (60 dias pós-parto), observou-se ganho de peso para todas as categorias, o que permitiu que chegassem ao final da estação de monta com peso vivo superior ao registrado no parto mantendo a diferença de peso entre as categorias. Dessa forma, vacas velhas e adultas (484,14 e 471,99 $\mathrm{kg}$, respectivamente) apresentaram peso superior $(\mathrm{P}<0,05)$ comparado às jovens $(414,43 \mathrm{~kg})$.

Foi verificada perda de peso $(\mathrm{P}>0,05)$ para todas as categorias no período compreendido entre o final da estação de monta e o desmame $(-17,96 \mathrm{~kg}$ para as adultas; -15,03 kg para as jovens e -13,55 kg para as velhas; Tabela 1). A perda de peso a partir dos 150 dias pós- parto pode ser reflexo da menor disponibilidade e qualidade da forragem, já que os animais foram encaminhados à pastagem predominantemente de Hemárthria e campo nativo no final do período reprodutivo.

A oferta de forragem com boa disponibilidade e qualidade durante a estação de parição de monta foi suficiente para manter um ganho de peso satisfatório (acima de $40 \mathrm{~kg}$ ) durante o período reprodutivo para todas as categorias, e com isso, possibilitou todas as categorias a alcançar em alta taxa de prenhez (Tabela 1), sendo $93 \%$ para vacas jovens, 94\% para vacas velhas e 96\% para adultas). Pötter e Lobato (2004) atribuem a alta taxa de prenhez em vacas primíparas (>90\%) a carga animal moderada e a condição corporal ao parto e ao início do acasalamento acima de 3,00 pontos.

Vacas que perdem condição corporal durante a lactação tem um intervalo prolongado do parto ao estro (CICCIOLI et al., 2012), no presente trabalho isso não ocorreu, sendo o período para a concepção em média de 44 dias após o início da estação de monta. O menor escore de condição corporal $(\mathrm{P}<0,05)$ das vacas jovens $(3,05$ pontos $)$ em relação às velhas $(3,24$ pontos $)$ no início da estação de monta não afetou o período de serviço, evidenciando que a manutenção do ECC na entrada na estação de monta acima de 3,00 pontos é suficiente para que vacas jovens alcancem intervalo entre o parto e a concepção similar às vacas adultas e velhas. Segundo Shrestha et al. (2005), o atraso da ovulação e prolongamento do período luteal são decorrentes de redução maior ou igual a uma unidade de ECC após o parto, o que não ocorreu no presente trabalho $(-0,20$ a $-0,31$ pontos; Tabela 1$)$.

O índice de produção de bezerro ao desmame por vaca (Tabela 2) acompanhou o peso do bezerro ao desmame (Tabela 3) sendo o menor índice para vacas jovens $(183,29 \mathrm{~kg})$, seguidas das velhas $(199,38 \mathrm{~kg})$ e adultas, as quais apresentaram a maior média $(214,57 \mathrm{~kg})$. Portanto, ao se avaliar a eficiência produtiva e reprodutiva observa-se que o índice de produção de bezerros por vaca não interferiu, passando as vacas jovens a apresentarem eficiência produtiva $(49,11 \mathrm{~kg})$ e reprodutiva $(47,64$ $\mathrm{kg})$ ao desmame similar às vacas adultas $(49,85 \mathrm{e}$ $48,35 \mathrm{~kg}$ ).

Já as vacas velhas, apresentaram menor $(\mathrm{P}<0,05)$ eficiência produtiva $(44,32 \mathrm{~kg})$ e reprodutiva $(42,99 \mathrm{~kg})$ mesmo com um intermediário peso de bezerro ao desmame. Isso se deve ao fato de que essa categoria apresentou o com maior peso vivo ao desmame $(470,59 \mathrm{~kg})$ em relação às vacas adultas $(451,92 \mathrm{~kg}$,) e jovens $(399,40 \mathrm{~kg}$; Tabela 2), sendo assim, quanto maior o peso vivo da vaca, maior deve ser o peso do bezerro ao desmame, evidenciando-se que apenas o peso ao desmame não deve ser tomado como referência única para se medir a eficiência produtiva do plante de cria. 
Tabela 2. Médias para eficiência produtiva e reprodutiva, erro padrão, coeficiente de variação $(\mathrm{CV})$ e probabilidade $(\mathrm{p}>\mathrm{F})$ para vacas de corte de diferentes categorias.

\begin{tabular}{lccccc}
\hline \multirow{2}{*}{ Características } & \multicolumn{3}{c}{ Categoria da vaca } & \multirow{2}{*}{ CV } & \multirow{2}{*}{$\operatorname{Pr}>$ F } \\
\cline { 2 - 5 } & Jovens & Adultas & Velhas & & \\
\hline $\begin{array}{l}\text { Índice de produção de bezerro ao } \\
\text { desmame (kg de bezerro por vaca-1 })^{1}\end{array}$ & $183,29 \pm 3,90^{\mathrm{c}}$ & $214,57 \pm 3,71^{\mathrm{a}}$ & $199,38 \pm 3,40^{\mathrm{b}}$ & 12,74 & $<.0001$ \\
$\begin{array}{l}\text { Eficiência produtiva ao desmame }(\mathrm{kg} \\
\text { de bezerro por 100kg de PVD } \text { PVD }^{2}\end{array}$ & $49,11 \pm 1,14^{\mathrm{a}}$ & $49,85 \pm 1,07^{\mathrm{a}}$ & $44,32 \pm 0,99^{\mathrm{b}}$ & 15,27 & 0,0333 \\
$\begin{array}{l}\text { Eficiência reprodutiva }\left(\mathrm{kg} \mathrm{de} \mathrm{bezerro}^{2}\right. \\
\left.\text { ao desmame por 100 } \mathrm{kg} \mathrm{de} \mathrm{PVD}^{-1}\right)^{3}\end{array}$ & $47,64 \pm 1,10^{\mathrm{a}}$ & $48,35 \pm 1,04^{\mathrm{a}}$ & $42,99 \pm 0,96^{\mathrm{b}}$ & 15,26 & 0,0333 \\
\hline
\end{tabular}

${ }^{1}=(\mathrm{PBD} \times \mathrm{TP}) / 100 .{ }^{2}=(\mathrm{PBD} / \mathrm{PVD}) \times 100 .{ }^{3}=(\mathrm{PBD} / 100) \times 100 \times \mathrm{TP} / \mathrm{PVD} . \mathrm{TP} . \mathrm{PBD}$, peso do bezerro ao Desmame $(\mathrm{kg}) ; \mathrm{TP}$, taxa de prenhez (\%); PVD, peso da vaca ao desmame $(\mathrm{kg})$.

Médias seguidas de letras diferentes na mesma linha diferem entre si pelo teste de Tukey $(\mathrm{P}<0,05)$.

Fonte: Elaboração dos autores.

Tabela 3. Médias de desempenho, erro padrão, coeficiente de variação (CV) e probabilidade (p>F) para bezerros em amamentação filhos de vacas de diferentes categorias.

\begin{tabular}{lccccc}
\hline \multirow{2}{*}{ Características } & \multicolumn{3}{c}{ Categoria das vacas } & \multirow{2}{*}{ CV } & \multirow{2}{*}{$\operatorname{Pr}>\mathrm{F}$} \\
\cline { 2 - 5 } & Jovens & Adultas & Velhas & & \\
\hline Peso ao nascer, kg & $31,96 \pm 0,82^{\mathrm{c}}$ & $37,98 \pm 0,74^{\mathrm{a}}$ & $35,42 \pm 0,72^{\mathrm{b}}$ & 14,94 & $<.0001$ \\
Ganho de peso do nascimento ao IEM, kg & $41,86 \pm 2,07^{\mathrm{b}}$ & $54,40 \pm 1,96^{\mathrm{a}}$ & $54,10 \pm 1,81^{\mathrm{a}}$ & 26,43 & 0,0102 \\
GMD do nascimento ao IEM, kg & $0,69 \pm 0,02^{\mathrm{b}}$ & $0,81 \pm 0,02^{\mathrm{a}}$ & $0,75 \pm 0,02^{\mathrm{a}}$ & 21,13 & 0,0044 \\
Peso bezerro no IEM, kg & $75,72 \pm 2,10^{\mathrm{b}}$ & $92,12 \pm 1,99^{\mathrm{a}}$ & $89,60 \pm 1,83^{\mathrm{a}}$ & 15,92 & $<.0001$ \\
GMD durante a estação de monta, kg & $0,71 \pm 0,02^{\mathrm{b}}$ & $0,88 \pm 0,02^{\mathrm{a}}$ & $0,76 \pm 0,02^{\mathrm{b}}$ & 17,86 & $<.0001$ \\
Ganho de peso total durante a EM, kg & $60,81 \pm 1,90^{\mathrm{b}}$ & $74,87 \pm 1,84^{\mathrm{a}}$ & $64,75 \pm 1,66^{\mathrm{b}}$ & 17,84 & $<.0001$ \\
Peso do bezerro ao FEM, kg & $136,54 \pm 3,17^{\mathrm{c}}$ & $165,90 \pm 3,01^{\mathrm{a}}$ & $154,25 \pm 2,76^{\mathrm{b}}$ & 13,57 & $<.0001$ \\
GMD do FEM ao desmame, kg & $0,93 \pm 0,03$ & $0,87 \pm 0,02$ & $0,81 \pm 0,02$ & 19,85 & 0,7700 \\
Ganho de peso total do FEM ao desmame, $\mathrm{kg}$ & $58,65 \pm 1,77$ & $55,30 \pm 1,69$ & $51,20 \pm 1,54$ & 19,97 & 0,8438 \\
Peso do bezerro ao desmame, kg & $190,00 \pm 3,54^{\mathrm{c}}$ & $221,20 \pm 3,37^{\mathrm{a}}$ & $205,19 \pm 3,09^{\mathrm{b}}$ & 11,14 & $<.0001$ \\
\hline
\end{tabular}

IEM = início da estação de monta; FEM = final da estação de monta; GMD = ganho médio diário. Médias seguidas por letras diferentes na mesma linha diferem estatisticamente entre si pelo teste de Tukey $(\mathrm{P}<0,05)$.

Fonte: Elaboração dos autores.

A menor média $(\mathrm{P}<0,05)$ de peso dos bezerros ao nascimento foi observada para os filhos de vacas jovens $(31,96 \mathrm{~kg})$ e as vacas adultas apresentaram os bezerros mais pesados $(37,98 \mathrm{~kg}$; Tabela 3$)$. Até os 60 dias de idade a progênie de vacas velhas tiveram desempenho similar às progênies de vacas adultas (54,40 e 54,10 kg, respectivamente), sendo superiores $(\mathrm{P}<0,05)$ ao desempenho dos filhos das vacas jovens $(41,86 \mathrm{~kg}$; Tabela 3$)$, os quais apresentaram no início da estação reprodutiva $18,30 \mathrm{~kg}$ a menos que os bezerros de vacas adultas e $15,78 \mathrm{~kg}$ a menos que bezerros filhos de vacas velhas.

Durante o período de maior exigência de leite pelo bezerro, Pimentel et al. (2006) encontraram uma produção de 2,46 L de leite a menos no pico de lactação de primíparas em relação às multíparas (8,27L vs. 5,81L), explicando desta forma o menor desempenho de bezerros filhos de vacas jovens. No presente trabalho, observamos que a partir dos 60 dias de idade, ou seja, durante o período de estação de monta, os bezerros filhos de vacas jovens 
passaram a apresentar o mesmo ganho de peso que os filhos de vacas velhas $(60,81$ e $64,94 \mathrm{~kg}$; Tabela 3), o que pode ser explicado pelo maior consumo de forragem pelo bezerro estimulado pela menor produção de leite da mãe (RESTLE et al., 2004) e à menor dependência do leite para a manutenção do bezerro com o decorrer da idade (PIMENTEL et al., 2006).

Os bezerros filhos de vacas adultas apresentaram o maior desempenho em todas as etapas de avaliação até 150 dias, chegando ao final do período reprodutivo com aproximadamente $30 \mathrm{~kg}$ e 11,65 $\mathrm{kg}$ a mais que os bezerros filhos de vacas jovens e velhas, respectivamente (Tabela 3). Este fato de maior desempenho de bezerros filhos de vacas adultas pode ser explicado pela maior produção de leite por vacas desta categoria. Restle et al. (2003), avaliando vacas em pastagem cultivada de inverno, observou que vacas adultas apresentaram produção de leite $12,2 \%$ superior $(5,61 \mathrm{~L} /$ dia $)$ às jovens $(5,00 \mathrm{~L} /$ dia $)$ e estas $24,6 \%$ superior em relação às velhas $(3,77 \mathrm{~L} / \mathrm{dia})$. Restle et al. (2004) relataram que a produção média diária de leite das vacas foi responsável por $45 \%$ do peso à desmama e $38 \%$ do ganho de peso dos bezerros.

A partir do período reprodutivo, ou seja, 150 dias de idade, a categoria da vaca não influenciou no ganho de peso médio diário, nem no ganho de peso total até o desmame aos sete meses, indicando o menor efeito da amamentação sobre os bezerros nesse período. Segundo Pimentel et al. (2006), o leite fornece $39 \%$ da exigência de energia líquida de ganho do bezerro aos 84 dias (equivalente à idade média dos bezerros no início da estação de monta no presente trabalho), mas não fornece energia para ganho aos 147 dias. Portanto, após 150 dias o bezerro supre suas demandas energéticas de ganho exclusivamente de alimentos sólidos e a capacidade da vaca em produzir leite não interfere mais no desempenho dos mesmos. Vale ressaltar que a época de nascimento e de reprodução utilizadas no presente trabalho permitiu uma boa eficiência reprodutiva das vacas e um bom desempenho ponderal dos bezerros.

Segundo Cerdótes et al. (2004), a menor produção de leite de vacas jovens é consequência do seu crescimento, pois, além dos nutrientes necessários para mantença e lactação, ainda necessitam de nutrientes para completar seu desenvolvimento. Já o melhor desempenho dos bezerros filhos de vacas adultas e velhas no pré-desmame é resultado das melhores condições fisiológicas desses animais em relação às jovens, resultando em maior produção de leite. Porém, o menor desempenho dos bezerros filhos de vacas velhas em relação às vacas adultas até o final do período reprodutivo (154,25 contra $165,90 \mathrm{~kg}$, respectivamente) pode ser atribuído ao declínio da produção de leite a partir de 6-7 anos de idade (SOUZA et al., 1996).

Evidencia-se então no presente trabalho que a partir dos 150 dias de idade a categoria da vaca não influenciou no desempenho dos bezerros (Tabela 3), e a diferença no peso ao desmame aos 7 meses de idade é decorrente da diferença do peso ao nascer e do desempenho até os 150 dias de idade. Desta forma, é de extrema importância a manutenção de boas condições nutricionais de vacas de corte entre os 90 e 120 dias pós-parto, pois, mesmo com o fornecimento de pastagem de baixa disponibilidade e qualidade a partir deste período (terceiro mês de estação de monta), as vacas desmamaram bezerros com peso relativamente alto aos sete meses (média de 205,46 kg de peso vivo entre as três classes de vacas avaliadas).

Assim, conclui-se que a taxa de prenhez do rebanho de cria não sofre influência da categoria das vacas de corte quando mantidas em boas condições corporais e nutricionais ao parto e durante a estação de monta. Nessas circunstâncias, vacas jovens acasaladas aos dois anos de idade apresentam eficiência produtiva e reprodutiva semelhante a de vacas adultas, enquanto a categoria de vacas velhas apresenta a menor eficiência reprodutiva do plantel de cria. 


\section{Referências}

CERDÓTES, L.; RESTLE, J.; ALVES FILHO, D. C.; NÖRNBERG, M. F. B. L.; NÖRNBERG, J. L.; HECK, I.; SILVEIRA, M. F. da. Produção e composição do leite de vacas de quatro grupos genéticos submetidas a dois manejos alimentares no período de lactação. Revista Brasileira de Zootecnia, Viçosa, MG, v. 33, n. 3, p. 610622, 2004.

CICCIOLI, N. H.; WETTEMANN, R. P.; SPICER, L. J.; LENTS, C. A.; WHITE, F. J.; KEISLER, D. H. Influence of body condition at calving and postpartum nutrition on endocrine function and reproductive performance of primiparous beef cows. Journal of Animal Science, Champaign, v. 81, n. 12, p. 3107-3120, 2003.

LOWMAN, B. G. Condition scoring beef cattle. Edinburgh: East of Scotland College of Agriculture, 1973. $8 \mathrm{p}$.

MORRIS, S. T.; MOREL, P. C. H.; KENYON, P. R. The effect of individual liveweight and condition of beef cows on their reproductive performance and birth and weaning weights of calves. New Zealand Veterinary Journal, London, v. 54, n. 2, p. 96-100, 2006.

OLIVEIRA, V. S. A.; BONATO, G. L.; SANTOS, R. M. Eficiência reprodutiva de vacas primíparas da raça Nelore. Acta Scientiae Veterinariae, Porto Alegre, v. 39, n. 2, p. 963-967, 2011.

PIMENTEL, M. A.; MORAIS, J. C. F.; JAUME, C. M.; LEMES, J. S.; BRAUNER, C. C. Características da lactação de vacas Hereford criadas em um sistema de produção extensivo na região da campanha do Rio Grande do Sul. Revista Brasileira de Zootecnia, Viçosa, MG, v. 35, n. 1, p. 159-168, 2006.

PÖTTER, B. A. A.; LOBATO, J. P. Efeitos de carga animal, pastagem melhorada e da idade de desmame no comportamentoreprodutivo de vacas primíparas. Revista Brasileira de Zootecnia, Viçosa, MG, v. 33, n. 1, p. $192-$ 202, 2004.

RENQUIST, B. J.; OLTJEN, J. W.; SAINZ, R. D.; CALVERT, C. C. Effects of age on body condition and production parameters of multiparous beef cows. Journal of Animal Science, Champaign, v. 84, n. 7, p. 1890-1895, 2006.
RESTLE, J.; PACHECO, P. S.; PASCOAL, L. L.; PÁDUA, J. T.; MOLETTA, J. L.; FREITAS, A. K. de.; LEITE, D. T. Efeito da pastagem, da produção e da composição do leite no desempenho de bezerros de diferentes grupos genéticos. Revista Brasileira de Zootecnia, Viçosa, MG, v. 33, n. 3, p. 691-703, 2004.

RESTLE, J.; PACHECO, P. S.; MOLETTA, J. L.; BRONDANI, I. L.; CERDÓTES, L. Grupo genético e nível nutricional pós-parto na produção e composição do leite de vacas de corte. Revista Brasileira de Zootecnia, Viçosa, MG, v. 32, n. 3, p. 585-597, 2003.

ROVIRA, J. Manejo nutritivo de los rodeos de cria em pastoreio. Montevideo: Hemisferio Sur, 1996. 288 p.

SHRESTHA, H. K.; NAKAO, T.; SUZUKI, T.; AKITA, M.; HIGAKI, T. Relationship between body condition score, body weight, and some nutritional parameters in plasma and resumption of ovarian cyclicity postpartum during pre-service period in high-producing dairy cows in a subtropical region in Japan. Theriogenology, Philadelphia, v. 64, n. 4, p. 855-866, 2005.

SOUZA, E. M.; MILAGRES, J. C.; REGAZZI, A. J.; MARTINEZ, M. L.; SILVA, M. de. A. Efeitos de fatores genéticos e de meio ambiente sobre a produção de leite em rebanhos de Gir leiteiro. Revista Brasileira de Zootecnia, Viçosa, MG, v. 25, n. 4, p. 889-901, 1996.

STATISTICAL ANALYSIS SYSTEM - SAS. System for microsoft windows: release 8.2. Cary, 2001. CD-ROM.

VAZ, R. Z.; LOBATO, J. F. P.; RESTLE, J. Productivity and efficiency of cow herds submitted to two weaning ages. Revista Brasileira de Zootecnia, Viçosa, MG, v. 39, n. 8, p. 1849-1856, 2010.

VIEIRA, A.; LOBATO, J. F. P.; CORREA, E. S.; TORRES JÚNIOR, R. A de A.; CEZAR, I. M. Produtividade e eficiência de vacas nelore em pastagem de Brachiaria decumbens Stapf nos cerrados do Brasil Central. Revista Brasileira de Zootecnia, Viçosa, MG, v. 34, n. 4, p. 13571365, 2005. 